\title{
Unrestricted generation of pure two-qubit states and entanglement diagnosis by single-qubit tomography
}

\author{
J. Gonzales ${ }^{1}$, P. Sánchez ${ }^{1}$, F. Auccapuclla ${ }^{1}$, B. Miller ${ }^{2}$, M. V. Andrés ${ }^{3}$, And F. De Zela ${ }^{1}$, \\ ${ }^{1}$ Departamento de Ciencias, Sección Física, Pontificia Universidad Católica del Perú, Apartado 1761, Lima, Peru. \\ ${ }^{2}$ Department of Physics, University of California, Santa Barbara, California 93106, USA \\ ${ }^{3}$ Departamento de Física Aplicada y Electromagnetismo-ICMUV, Universidad de Valencia, c/Dr. Moliner 50, Burjassot, Valencia, Spain \\ *Corresponding author: fdezela@pucp.edu.pe
}

\begin{abstract}
We present an experimental proof-of-principle for the generation and detection of pure two-qubit states which have been encoded in degrees of freedom that are common to both classical-light beams and single photons. Our protocol requires performing polarization tomography on a single qubit from a qubit pair. The degree of entanglement in the qubit pair is measured by concurrence, which can be directly extracted from intensity measurements - or photon counting - entering single-qubit polarization tomography.
\end{abstract}

Entangled qubit pairs are basic units in schemes devised to implement quantum information processes such as quantum communication, quantum cryptography, etc., as well as in schemes designed to address foundational issues of quantum mechanics. The exploitation of entanglement is one of the most challenging goals of quantum information technologies. There are good reasons to believe that entanglement plays a key role in the advantage that quantum circuits would have over classical circuits [1]. Entanglement is however difficult to characterize experimentally. So-called entanglement witnesses are state specific, in the sense that they are tailored to detecting some types of entanglement while they are blind to others. Alternatively, one can rely on entanglement measures, which are designed to be state independent. A prominent example is concurrence, which is defined for any pure, bipartite state $\Phi$ as $C(\Phi)=\left|\left\langle\Phi\left|\left(\sigma_{y} \otimes \sigma_{y}\right)\right| \Phi^{*}\right\rangle\right|$, where $\sigma_{y}$ is the Pauli matrix and $\left|\Phi^{*}\right\rangle$ the complex-conjugate of $|\Phi\rangle$ in the computational basis of the tensor-product space to which $\Phi$ belongs. Now, confronted with this measure, the experimentalist sees no obvious way to implement it directly in the laboratory. To begin with, complex conjugation is an unphysical operation, because it does not conserve positive-definiteness. Thus, the only way to obtain $C(\Phi)$ from measurements seems to be by means of full tomographic determination of state $\Phi$, which is experimentally demanding and prone to inaccuracies. The evaluation of $C(\Phi)$, which nonlinearly depends on the parameters fixing $\Phi$, can then be too inaccurate.

Back in 2005, Mintert et al. [2] found a way out of the above problem, a way that circumvented the need of performing quantum state tomography. They succeeded in re-writing $C(\Phi)$ as an expectation value of a Hermitian operator: $C(\Phi)=$ $(\langle\Phi|\otimes\langle\Phi|A| \Phi\rangle \otimes| \Phi\rangle)^{1 / 2}$, thereby showing how to get $C$ by projective measurements. As we see, one must deal with a twofold copy of $|\Phi\rangle$. Walborn et al. [3] provided a proof-ofprinciple, which was also the first direct experimental observation of an entanglement measure. They addressed bipartite states that were physically realized by single photons carrying two degrees of freedom (DOFs), polarization and momentum (or path). Thus, $|\Phi\rangle \in \mathcal{H}_{\text {pol }} \otimes \mathcal{H}_{\text {path }} \equiv \mathcal{H}$, so that $A$ acts on $\mathcal{H} \otimes \mathcal{H}$. While the approach of Walborn et al. introduces the difficulty of preparing each time two copies of $\Phi$ to get $C(\Phi)$, only a one-setting measurement is needed. This is so because $A=4 P_{-}^{(1)} \otimes P_{-}^{(2)}$, where $P_{-}^{(j)}$ is the projector onto the antisymmetric part of the $j$-th subsystem [4]. It can be readily shown that the expectation value of $P_{-}^{(1)} \otimes P_{-}^{(2)}$ on a twofold copy of $|\Phi\rangle$ gives the same result as the expectation value of, say, $\mathbf{1}^{(1)} \otimes P_{-}^{(2)}$, so that only the second subsystem has to be projected. In particular, for a two-state system, $P_{-}$is the projector onto the singlet state. In the case of Walborn et al., the polarization subspace is spanned by horizontally and vertically polarized states, $|h\rangle$ and $|v\rangle$, respectively. The binary path-DOF is spanned by analogous, mutually orthogonal vectors, $|x\rangle$ and $|y\rangle$, say. $C(\Phi)$ can then be determined by measuring the probability to detect one photon of the photon-pair $|\Phi\rangle|\Phi\rangle$ in the singlet state $\left|\Psi^{-}\right\rangle=(|h\rangle|y\rangle-|v\rangle|x\rangle) / \sqrt{2}$. The other photon serves only as a trigger for the detection of its twin.

A theoretical proposal to overcome the disadvantage of requiring two copies of the same state was made by Fei et al. [5]. Their proposal derives from writing $C(\Phi)$ in terms of the identity matrix $\sigma_{0}$ and the Pauli matrices as

$$
\begin{aligned}
2 C^{2}(\Phi) & =1+\left\langle\sigma_{3} \sigma_{3}\right\rangle^{2}-\left\langle\sigma_{3} \sigma_{0}\right\rangle^{2}-\left\langle\sigma_{0} \sigma_{3}\right\rangle^{2} \\
& -\left\langle\sigma_{0} \sigma_{1}\right\rangle^{2}+\left\langle\sigma_{3} \sigma_{1}\right\rangle^{2}-\left\langle\sigma_{0} \sigma_{2}\right\rangle^{2}+\left\langle\sigma_{3} \sigma_{2}\right\rangle^{2}
\end{aligned}
$$

Here, expectation values refer to the bipartite state $\Phi$ alone. However, in this case, as in the case of Walborn et al., while only a one-setting measurement is required, it involves a nonlocal projection on two entangled DOFs. Projections of this kind are rather involved. They are done by means of, e.g. 
controlled-NOT gates, which are relatively sophisticated arrangements. Recently, a proposal was made that avoids the need of controlled-NOT gates [6]; but it is again a two-copybased protocol.

In this Letter, we present a protocol to measure $C(\Phi)$ without having to produce a copy of $\Phi$. Moreover, the measurement of $C(\Phi)$ involves only one of the two DOFs that $\Phi$ carries along. Thus, only local projections are required. In addition, our scheme also serves to perform accurate state tomography. The scheme is based on a newly established relationship [7] between concurrence and the degree of polarization $P$ of a twostate system: $C^{2}+P^{2}=1$. This result reduces the task of getting $C$ to the task of getting $P$, for which we can apply standard techniques. For comparison, see Ref. [8], where $C^{2}+P^{2}=1$ was also invoked.

For the general two-qubit pure state

$\left|\Phi_{A B}\right\rangle=r_{1} e^{i \phi_{1}}|h\rangle|x\rangle+r_{2} e^{i \phi_{2}}|v\rangle|x\rangle+r_{3} e^{i \phi_{3}}|h\rangle|y\rangle+r_{4} e^{i \phi_{4}}|v\rangle|y\rangle$,

the above relationship reads [9]:

$$
C^{2}+P^{2}=\left(\sum_{k=1}^{4} r_{k}^{2}\right)^{2}=1,
$$

where we have assumed $\left\langle\Phi_{A B} \mid \Phi_{A B}\right\rangle=1$, without loss of generality. The degree of polarization $P$ is common to the two reduced density matrices that are associated to state $\left|\Phi_{A B}\right\rangle$, namely $\rho_{A}=\operatorname{Tr}_{B}\left|\Phi_{A B}\right\rangle\left\langle\Phi_{A B}\right|$ and $\rho_{B}=\operatorname{Tr}_{A}\left|\Phi_{A B}\right\rangle\left\langle\Phi_{A B}\right|$. These $2 \times 2$ matrices can be written in terms of the unit matrix $\sigma_{0}$ and the Pauli matrices $\sigma=\left(\sigma_{1}, \sigma_{2}, \sigma_{3}\right)$, in the form

$$
\rho_{k}=\frac{1}{2}\left(\sigma_{0}+\mathbf{S}_{(k)} \cdot \sigma\right) \equiv \frac{1}{2}\left(\sigma_{0}+P \hat{\mathbf{n}}_{(k)} \cdot \sigma\right),
$$

where $\mathbf{S}_{(k)}=\operatorname{Tr}_{k}\left(\rho_{k} \sigma\right)(k=A, B)$ are two Stokes vectors and $P$ their common modulus: $P=\sqrt{S_{(k) 1}^{2}+S_{(k) 2}^{2}+S_{(k) 3}^{2}}$. Hence, concurrence is given by

$$
C=\sqrt{1-\mathbf{S}_{(A)} \cdot \mathbf{S}_{(A)}}=\sqrt{1-\mathbf{S}_{(B)} \cdot \mathbf{S}_{(B)}} .
$$

If we can perform state tomography of $\left|\Phi_{A B}\right\rangle$, then we can construct $\rho_{A}$ and /or $\rho_{B}$ and from them $\mathbf{S}_{(A)}$ and/or $\mathbf{S}_{(B)}$ to obtain $C$ by means of equation (5). As we shall see, we can indeed accurately characterize general two-qubit states $\left|\Phi_{A B}\right\rangle$ by performing only single-qubit measurements.

As recently shown, eleven is the minimum number of Pauli measurements needed to determine a two-qubit pure state [10]. Previously, it was shown that at least sixteen Pauli measurements are needed in the case of mixed states [11]. Pauli measurements provide expectation values of Pauli operators. A protocol originally proposed by James et al. [11], which is based on Pauli measurements, soon became very popular in optical experiments despite some drawbacks. Indeed, said protocol has been shown to be biased: it can give rise to a systematic underestimation of fidelity [12], which is perhaps the most commonly employed figure of merit in quantum state tomography (QST). Furthermore, by proper choice of projective measurements, the average fidelity can be optimized. The protocol of Ref. [11] performs rather poorly in comparison to others that use projective measurements along directions defined by Platonic solids [13]. Fixing the set of measured observables amounts to fixing their orthonormal eigenbases. How many of these are required to distinguish all pure states is a question that was recently answered for all Hilbert-space dimensions $d \geq 2$, with exception of $d=4$ [14]. The minimal number of orthonormal bases that can distinguish all pure states is three for $d=2$; it is four for $d=3$ and $d \geq 5$, whereas for $d=4$ it may be either three or four [14]. Results of this type are important to guide experimental implementations of QST. Naturally, one strives to reduce the experimental requirements as much as possible. It could be advantageous to perform more projections than the minimum, whenever one projects on product states instead of entangled states. Ease of manipulation is also important, together with scalability. Our procedure requires Pauli measurements on one qubit at a time, plus one interferometric record to fix a relative phase. The number of measurements depends on the measured state. Our protocol is thus of the adaptive type [15, 16], much in the spirit of a very recent theoretical proposal [17]. In contrast to other layouts (see, e.g., [18]), ours does not require several phase shifters to deal with the binary path DOF. Only standard half- and quarter-wave plates, one piezo-transducer and beam splitters are necessary to deal with both preparation and tomographic characterization of general, two-qubit pure states. We performed our experiments using classical light, as generation of pure states is best accomplished using coherent light beams. Single photons introduce unavoidable noise that makes the generated states depart from the ideal, target pure state. It was therefore advisable to test the feasibility of our scheme by applying it first to coherent laser light. Performing QST-related tests with classical light is becoming a standard approach [19]. There is a growing realization that non-separability is not restricted to quantum entanglement [19]. Hence, quantum features that stem from non-separability alone may have a counterpart in classical light. Using it, one can avoid several quantum measurement woes. As long as quantum non-locality is not involved in the tested features, a classical test may perfectly mirror the quantum version. Toninelli et al. [19] have recently provided up-to-date information about QST-related tests with classical light.

Our procedure serves to generate general two-qubit states of the form given by Eq. (2). In what follows, we use the shorthand notation $|h, x\rangle$ instead of $|h\rangle|x\rangle$, etc. Without loss of generality, we may take $\sum_{i=1}^{4} r_{i}^{2}=1$ and add to $\left|\Phi_{A B}\right\rangle$ an arbitrary global phase factor, which is equivalent to set, say, $\phi_{1}=0$. Accordingly, we may use the convenient parametrization

$$
\begin{array}{ll}
r_{1}=\cos \theta \cos \theta_{x}, \quad \phi_{1}=0 ; & r_{2}=\cos \theta \sin \theta_{x}, \quad \phi_{2}=\phi_{x} \\
r_{3}=\sin \theta \cos \theta_{y}, \quad \phi_{3}=\delta ; & r_{4}=\sin \theta \sin \theta_{y}, \quad \phi_{4}=\delta+\phi_{y}
\end{array}
$$

Hence, $\left|\Phi_{A B}\right\rangle$ contains six independent parameters: $\theta, \theta_{x}, \theta_{y}, \delta$, $\phi_{x}$, and $\phi_{y}$. Our setup is shown in Fig. (1). By proper choice of the angles to which the wave plates are set, one can generate any two-qubit state of the form given by Eqs. (2) and (6). In other words, one can always solve - either analytically or numerically - the set of equations whose unknowns are the wave plate's angles $\alpha_{i}$ and $\beta_{i}$.

Our setup works as follows. The laser source delivers a horizontally polarized beam $|h\rangle$. A half-wave plate set to $\theta / 2$ produces the transformation $|h\rangle \rightarrow \cos \theta|h\rangle+\sin \theta|v\rangle$. At the output ports of the polarizing beam-splitter (PBS) we have states $|h, x\rangle$ and $|v, y\rangle$ with relative weights $\cos \theta$ and $\sin \theta$, respectively. A displaceable mirror introduces the relative phase $\delta$ between the two arms of the interferometer. In this way, state

$$
\left|\psi_{i}\right\rangle=\cos \theta|h, x\rangle+e^{i \delta} \sin \theta|v, y\rangle
$$

is prepared before the $Q H$ configurations $(Q=$ quarter-wave 


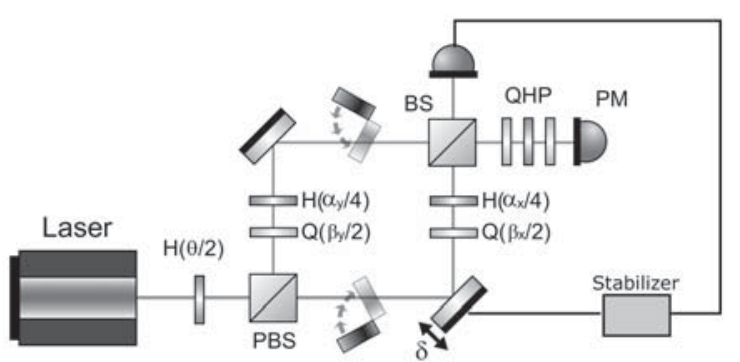

Fig. 1. Setup for state generation and tomography of twoqubit pure states. $H$ : half-wave plate; $Q$ : quarter-wave plate; $P B S$ : polarizing beam splitter; $P$ : polarizer; $B S$ : beam splitter; PM: photomultiplier. Stabilizer is an integral-control electronic circuit [20] which displaces a movable mirror that stabilizes the interferometer and fixes phase $\delta$.

plate, $H=$ half-wave plate) that are placed on each arm. This configuration can output any desired polarization state

$$
|\vartheta, \varphi\rangle_{\mathrm{pol}}=\cos \vartheta|h\rangle+e^{i \varphi} \sin \vartheta|v\rangle,
$$

out of a linearly polarized, input state [21]. We can achieve, for instance, that $H\left(\alpha_{x} / 4\right) Q\left(\beta_{x} / 2\right)|h\rangle=\left|\theta_{x}, \phi_{x}\right\rangle_{\text {pol }}$ and $H\left(\alpha_{y} / 4\right) Q\left(\beta_{y} / 2\right)|v\rangle=\left|\theta_{y}, \phi_{y}\right\rangle_{\text {pol }}$. Then, after the $Q H$ configurations that are placed on each arm of the arrangement (see Fig. (1)), the input state $\left|\psi_{i}\right\rangle$ will have been transformed into

$$
\begin{aligned}
\left|\psi_{f}\right\rangle & =\cos \theta \cos \theta_{x}|h, x\rangle+e^{i \delta} \sin \theta \cos \theta_{y}|h, y\rangle \\
& +\cos \theta \sin \theta_{x} e^{i \phi_{x}}|v, x\rangle+e^{i\left(\delta+\phi_{y}\right)} \sin \theta \sin \theta_{y}|v, y\rangle .
\end{aligned}
$$

This is the state just before the second beam-splitter of our setup. In this way, we generate the target state given by equations (2) and (6). The settings of the wave plates, $\alpha_{i=x, y}$ and $\beta_{i=x, y}$, satisfy the equations that result from equating the Stokes vectors associated to $H\left(\alpha_{x} / 4\right) Q\left(\beta_{x} / 2\right)|h\rangle$ and $\left|\theta_{x}, \phi_{x}\right\rangle_{\text {pol }}$, as well as those associated to $H\left(\alpha_{y} / 4\right) Q\left(\beta_{y} / 2\right)|v\rangle$ and $\left|\theta_{y}, \phi_{y}\right\rangle_{\text {pol }}$. We get

$$
\begin{aligned}
& \alpha_{x / y}=\tan ^{-1}\left(\cos \phi_{x / y} \tan \theta_{x / y}\right) \pm \sin ^{-1}\left(\sin \phi_{x / y} \sin \theta_{x / y}\right) \\
& \beta_{x / y}= \pm \sin ^{-1}\left(\sin \phi_{x / y} \sin \theta_{x / y}\right) .
\end{aligned}
$$

By slightly modifying the interferometric layout that generates two-qubit pure states, one can perform state tomography. This is done with a standard array that consists of a quarter-wave plate, a half-wave plate and a polarizer, which are mounted at one output-port of the beam-splitter (see Fig. (1)). Having characterized the state, one can straightforwardly obtain the fidelity (see below) as a figure of merit. Our tomographic protocol consists of the following steps, schematically shown in Fig. (2):

1. One obtains $r_{1}, r_{2}$ and $\phi_{2}$ by first blocking the $y$-arm of the interferometer to perform standard, single-qubit polarization tomography on the $x$-beam, thereby getting its Stokes vector. This yields $r_{1}|h, x\rangle+r_{2} e^{i \phi_{2}}|v, x\rangle$, up to a global phase factor.

2. Similarly to the first step, one now blocks the $x$-arm and performs single-qubit polarization tomography with the $y$ beam. This yields $r_{3} e^{i \phi_{3}}|h, y\rangle+r_{4} e^{i \phi_{4}}|v, y\rangle$, up to a global phase factor, i. e., we obtain $r_{3}, r_{4}$ and $\phi_{4}-\phi_{3}$.
3. After placing polarizers so as to project upon horizontally polarized states on both arms, one generates an interferometric pattern out of the two, equally polarized beams. This pattern has a shift $\phi_{3}$ with respect to a reference pattern that is previously produced using the stabilizing electronic circuit shown in figure (1).

The above method yields in fact the absolute value of $\phi_{3}$. Though it is possible to get its sign by first fixing the sign of the derivative in the stabilizing electronic circuit, in practice it was easier to determine the sign of $\phi_{3}$ by a two-step "trial and error" procedure. Thus, the fidelity calculation (see below) was performed twice, with $\pm \phi_{3}$. The value that was much closer to unity corresponded to the correct sign of $\phi_{3}$.

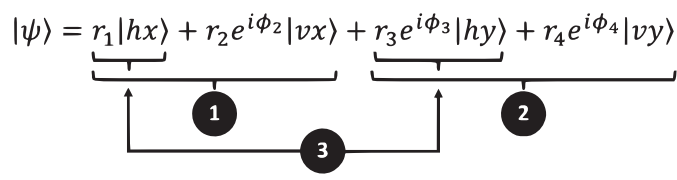

Fig. 2. Tomographic procedure. Steps 1 and 2 correspond to single-qubit polarimetric tomography, i.e., determination of Stokes vectors $\mathbf{S}_{x}^{(\mathrm{pol})}$ and $\mathbf{S}_{y}^{(\mathrm{pol})}$, respectively. Step 3 corresponds to interferometric measurement of phase $\phi_{3}$.

We used two kinds of states to perform a proof-of-principle of our scheme. To test the accuracy of our method, we used as a figure of merit the fidelity [22] of the experimentally produced state to the target state:

$$
F=\left|\left\langle\psi_{\text {target }} \mid \psi_{\exp }\right\rangle\right| \text {. }
$$

As we said, our target states were of two kinds. In each case, the states depended on a tunable parameter, the variation of which allowed us to change the degree of entanglement, i.e., concurrence $C \in[0,1]$, which for state $\left|\Phi_{A B}\right\rangle$ reads

$$
C\left(\Phi_{A B}\right)=|\sin 2 \theta|\left[\sin 2 \theta_{x} \sin 2 \theta_{y} \sin ^{2}\left(\frac{\phi_{x y}}{2}\right)+\sin ^{2} \theta_{x y}\right]^{1 / 2},
$$

with $\phi_{x y}=\phi_{x}-\phi_{y}$ and $\theta_{x y}=\theta_{x}-\theta_{y}$.

We first chose as target a state with amplitudes $r_{i}=1 / 2$, with all but one of its phases having a fixed value:

$$
|\Phi\rangle=\frac{1}{2}|h, x\rangle+\frac{1}{2}|h, y\rangle+\frac{1}{2} e^{i \pi / 2}|v, x\rangle+\frac{1}{2} e^{i(\phi+\pi / 2)}|v, y\rangle .
$$

By letting $\phi$ take the values $0^{\circ}, 45^{\circ}, 90^{\circ}, 135^{\circ}, 180^{\circ}$, our target state $|\Phi\rangle$ varied from being fully separable, to completely entangled. Equation (9) reduces to (12) by setting $\theta=\theta_{x}=\theta_{y}=$ $45^{\circ}, \phi_{x}=0^{\circ}, \delta=90^{\circ}$, while $\phi_{y}=\phi$ takes the aforementioned values. The corresponding wave plates' orientations $\alpha_{y}$ and $\beta_{y}$ are then readily obtained. The theoretical value of concurrence is given by

$$
C(\Phi)=\left|\sin \left(\frac{\phi}{2}\right)\right|
$$

Notice that it is possible to go from a factorable state to an entangled one, because the two-qubit state is subjected to a nonlocal unitary transformation: $U_{\text {path-pol }} \neq U_{\text {path }} \otimes U_{\text {pol }}$. That is, the polarization transformation depends on path (the $y$-arm in this case).

Our second target state was

$|\Gamma\rangle=\frac{\sqrt{3}}{2} \cos \gamma|h, x\rangle+\frac{\sqrt{3}}{2} e^{i \frac{\pi}{4}} \sin \gamma|v, x\rangle+\frac{i \sqrt{3}}{4}|h, y\rangle+\frac{1}{4} e^{i \frac{5 \pi}{8}}|v, y\rangle$. 
The fixed parameters in equation (9) are now $\theta=\theta_{y}=$ $30^{\circ}, \phi_{x}=45^{\circ}, \phi_{y}=22.5^{\circ}, \delta=90^{\circ}$. Two amplitudes periodically change with $\theta_{x}=\gamma$, as specified in (14). In this case, concurrence is theoretically given by

$$
C(\Gamma)=\frac{\sqrt{3}}{2}\left[\sin ^{2}\left(\frac{\pi}{6}-\gamma\right)+\frac{\sqrt{3}}{2} \sin ^{2}\left(\frac{\pi}{16}\right) \sin (2 \gamma)\right]^{1 / 2} .
$$

By applying our tomographic procedure, we determined that the actually produced states had fidelities ranging from 0.997 to 0.999 in the case of $\Phi$ and from 0.995 to 0.999 in the case of $\Gamma$.

Once we have performed state-tomography of $\left|\Phi_{A B}\right\rangle$, we can get $\mathbf{S}_{(A)}$ and/or $\mathbf{S}_{(B)}$ to obtain $C$ by means of equation (5). In our case, $\mathbf{S}_{(A)}=\mathbf{S}_{\text {pol }}$ and $\mathbf{S}_{(B)}=\mathbf{S}_{\text {path }}$. One can readily show that, for a Mach-Zehnder-type configuration such as the one we employ,

$$
\mathbf{S}_{\mathrm{pol}}=\left(\frac{I_{x}^{(h)}+I_{x}^{(v)}}{I_{\mathrm{tot}}}\right) \mathbf{S}_{x}^{(\mathrm{pol})}+\left(\frac{I_{y}^{(h)}+I_{y}^{(v)}}{I_{\mathrm{tot}}}\right) \mathbf{S}_{y}^{(\mathrm{pol})},
$$

with $I_{\text {tot }}=\left(I_{x}^{(h)}+I_{x}^{(v)}\right)+\left(I_{y}^{(h)}+I_{y}^{(v)}\right)$. The symbol $I_{x}^{(h)}$ refers to the intensity measured on the $x$-arm with a polarizer set to pass horizontally polarized light, and similarly for the other symbols. In (16), $\mathbf{S}_{x}^{(\mathrm{pol})}$ refers to the Stokes vector that is obtained by blocking the $y$-arm of the interferometer, and similarly $\mathbf{S}_{y}^{(\mathrm{pol})}$. With these measured vectors we obtain $\mathbf{S}_{\mathrm{pol}}$ via equation (16), and so we get $C$ using (5) with $\mathbf{S}_{(A)}=\mathbf{S}_{\text {pol }}$.

In summary, we have two ways by which we can obtain $C$ from our measurements. One way starts with the construction of $\left|\Phi_{A B}\right\rangle\left\langle\Phi_{A B}\right|$ from the tomographic data. Tracing $\left|\Phi_{A B}\right\rangle\left\langle\Phi_{A B}\right|$ over any one of the two degrees of freedom gives the corresponding Stokes vector entering equation (5). The other way is to obtain $\mathbf{S}_{\mathrm{pol}}$ from $\mathbf{S}_{x}^{(\mathrm{pol})}$ and $\mathbf{S}_{y}^{(\mathrm{pol})}$, as per equation (16). The two methods produce the same values of $C$, within the accuracy level of our plots, figures (3) and (4). We should notice that $\sum_{k=1}^{4} r_{k}^{2}$ was not exactly unity and thus we consistently used the relationship $C=\left[\left(\sum_{k=1}^{4} r_{k}^{2}\right)^{2}-\mathbf{S}_{\mathrm{pol}} \cdot \mathbf{S}_{\mathrm{pol}}\right]^{1 / 2}$ instead of equation (5), which holds for the ideal case. Our main error source came from the angular resolution of wave plates and polarizers $\left( \pm 1^{\circ}\right)$. We expect to have an additional error source coming from the unavoidable quantum noise in followup experiments with single photons. As the employed DOFs are common to both quantum and classical light, basically the same scheme can be used in the two cases.

\section{FUNDING INFORMATION}

This work was partially financed by DGI-PUCP (Grant No. 441). MVA acknowledges financial support from the Generalitat Valenciana of Spain (Grant BEST-2018-179).

\section{REFERENCES}

1. S. Bravyi, D. Gosset, R. König, Science 362, 308 (2018).

2. F. Mintert, M. Kus, and A. Buchleitner, Phys. Rev. Lett. 95, 260502 (2005).

3. S. P. Walborn, P. H. Souto Ribeiro, L. Davidovich, F. Mintert, and A. Buchleitner, Nature (London) 440, 1022 (2006).

4. S. P. Walborn, P. H. Souto Ribeiro, L. Davidovich, F. Mintert, and A Buchleitner, Phys. Re. A 75, 032338 (2007).

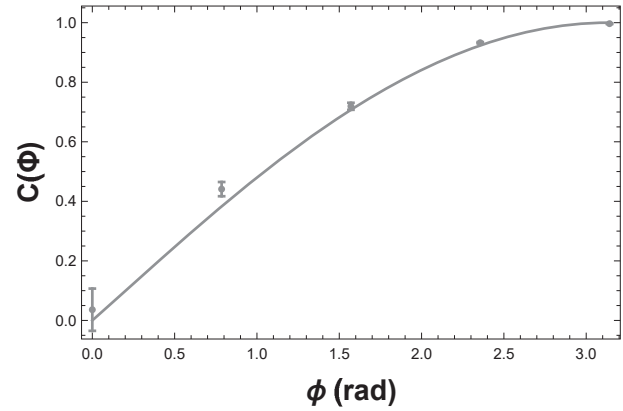

Fig. 3. Concurrence for state $|\Phi\rangle$ (see text). The solid curve is the theoretical prediction, given by equation (13). Parameter $\phi$ corresponds to a variable phase factor.

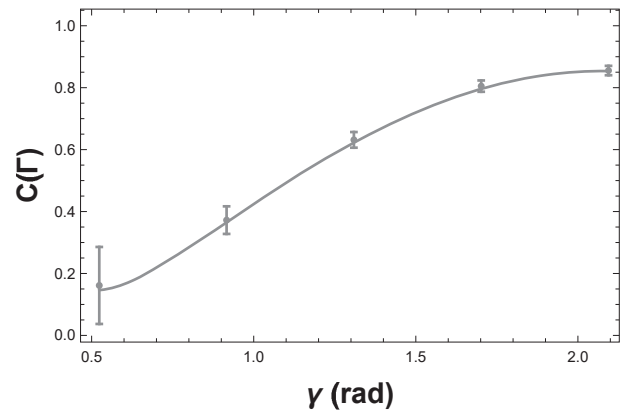

Fig. 4. Concurrence for state $|\Gamma\rangle$ (see text). The solid curve is the theoretical prediction, given by equation (15). Parameter $\gamma$ corresponds to variable amplitudes.

5. S-M. Fei, M-J. Zhao, K. Chen, and Z-X. Whang, "Experimental determination of entanglement for arbitrary pure states", Phys. Re. A $\mathbf{8 0}$, 032320 (2009).

6. T. Zeng, W-J. Chu, Q. Yang, M. Yang, W. Song, and Z-L. Cao, Quantum Inf. Process 16, 262 (2017).

7. X.-F. Qian, T. Malhotra, A. N. Vamivakas, and J. H. Eberly, Phys. Rev. Lett. 117, 153901 (2016).

8. X.-F. Qian, A. N. Vamivakas, and J. H. Eberly, Optica, 5, 942 (2018).

9. F. De Zela, Opt. Lett. 43, 2603 (2018).

10. X. Ma et al., Phys. Rev. A 93, 032140 (2016).

11. D. F. V. James, P. G. Kwiat, W. J. Munro, and A. G. White, Phys. Rev. A 64, 052312 (2001).

12. C. Schwemmer et al., Phys. Rev. Lett. 114, 080403 (2015).

13. M. D. de Burgh, N. K. Langford, A. C. Doherty, and A. Gilchrist, Phys. Rev. A 78, 052122 (2008).

14. C. Carmeli, T. Heinosaari, J. Schultz, and A. Toigo, Eur. Phys. J.D. 69, 179 (2015).

15. R. J. Chapman, C. Ferrie, and A. Peruzzo, Phys. Rev. Lett. 117, 040402 (2016).

16. D. Goyeneche, G. Cañas, S. Etcheverry. E. S. Gómez, G. B. Xavier, G. Lima, and A. Delgado, Phys. Rev. Lett. 115, 090401 (2015).

17. Y. Wang and Y. Shang, Chin. Phys. B 27, 100306 (2018).

18. B.-G. Englert, C. Kurtsiefer, and H. Weinfurter, Phys. Rev. A 63, 032303 (2001).

19. E. Toninelli, B. Ndagano, A. Vallés, B. Sephton, I. Nape, A. Ambrosio, F. Capasso, M. J. Padgett, and A. Forbes, Adv. Opt. Photonics 11, 67 (2019).

20. D. A. Jackson, R. Priest, A. Dandridge, A. B. Tveten, Appl. Opt. 19, 2926 (1980).

21. J. N. Damask, Polarization Optics in Telecommunications, Springer, New York, 2005

22. M. A. Nielsen and I. L. Chuang, Quantum Computation and Quantum Information, Cambridge University Press, Cambridge, 2007. 